\title{
miR-210 regulates vascular endothelial cell apoptosis in arteriosclerosis obliterans through the JAK1-STAT3 pathway
}

\section{Type}

Research paper

\section{Keywords}

Apoptosis, STAT3, miR-210, HUVECs cells, JAK1, arteriosclerosis obliterans

\begin{abstract}
Introduction

Atherosclerosis is a continuously worsening chronic condition that starts in the arteries and may then affect other blood vessels. There is increasing evidence linking microRNAs to the development of arteriosclerosis obliterans (ASO). Quantitative reverse transcription polymerase chain reaction (qRTPCR) was applied in this study to detect miR-210-5p (miR-210) expression, and significant upregulation was observed in human arterial walls where ASO was prevalent.

\section{Material and methods}

The proliferation of human umbilical vascular endothelial cells (HUVECs) with various levels of miR-210 expression was assessed via MTT and colony-formation assays. Cell proliferation was significantly promoted in HUVECs with upregulated miR-210 levels and reduced in HUVECs with downregulated miR-210 levels.

\section{Results}

Flow cytometric analysis of cells stained with annexin V-FITC and propidium iodide data demonstrated that miR-210 inhibited apoptosis, while miR-210 inhibition promoted apoptosis by mediating pro-apoptotic protein expression levels. These results were verified using a dual-luciferase reporter gene assay system, which showed that Janus kinase 1 (JAK1) was directly targeted by miR-210, while an miR-210 mimic significantly decreased downstream JAK1 and signal transducer and activator of transcription 3 (STAT3) activation at a post-transcriptional level in HUVECs, as detected by western blotting and qRT-PCR. Further inhibition of either JAK1 or STAT3 counteracted the effect of miR-210 on HUVEC proliferation and apoptosis. These findings suggest that miR-210 promotes HUVEC proliferation, at least in part, by targeting the JAK1-STAT3 signaling axis.
\end{abstract}

\section{Conclusions}

This study provides insights into the contribution of the miR-210-JAK1-STAT3 axis and its underlying mechanisms to ASO pathology. 
$m i R-210$ regulates vascular endothelial cell apoptosis in arteriosclerosis obliterans through the JAK1-STAT3 pathway

Songjie Hu, Zhongjian Xu, Dehai Lang*

Department of Vascular Surgery, Ningbo Huamei Hospital, Chinese Academy of Sciences, Ningbo, Zhejiang, China

Running head: $m i R-210$ regulates VEC apoptosis

*Address correspondence to: Dehai Lang, Department of Vascular Surgery, Ningbo Huamei Hospital, Chinese Academy of Sciences, No. 42, Yongfeng Road, Haishu District, Ningbo, 315012 Zhejiang, China

Phone: +86-0574-83870286

Email: dehailang@163.com

\section{Authors email}

Songjie $\mathrm{Hu}$

email: shenmeiho44140@163.com

Zhongjian Xu

email: pomian8324j@163.com

Dehai Lang

email: dehailang@163.com 


\section{Structured Abstract}

Introduction: Atherosclerosis is a continuously worsening chronic condition that starts in the arteries and may then affect other blood vessels. There is increasing evidence linking microRNAs to the development of arteriosclerosis obliterans (ASO). Quantitative reverse transcription polymerase chain reaction (qRT-PCR) was applied in this study to detect $m i R-210-5 p(m i R-210)$ expression, and significant upregulation was observed in human arterial walls where ASO was prevalent. Material and Methods: The proliferation of human umbilical vascular endothelial cells (HUVECs) with various levels of $m i R-210$ expression was assessed via MTT and colony-formation assays. Cell proliferation was significantly promoted in HUVECs with upregulated $m i R-210$ levels and reduced in HUVECs with downregulated miR-210 levels. Results: Flow cytometric analysis of cells stained with annexin V-FITC and propidium iodide data demonstrated that $m i R-210$ inhibited apoptosis, while $m i R-210$ inhibition promoted apoptosis by mediating pro-apoptotic protein expression levels. These results were verified using a dual-luciferase reporter gene assay system, which showed that Janus kinase 1 (JAK1) was directly targeted by $m i R-210$, while an $m i R-210$ mimic significantly decreased downstream JAK1 and signal transducer and activator of transcription 3 (STAT3) activation at a post-transcriptional level in HUVECs, as detected by western blotting and qRT-PCR. Further inhibition of either JAK1 or STAT3 counteracted the effect of $m i R-210$ on HUVEC proliferation and apoptosis. These findings suggest that $m i R-210$ promotes HUVEC proliferation, at least in part, by targeting the JAK1-STAT3 signaling axis. 
Discussion: This study provides insights into the contribution of the miR-210-JAK1-STAT3 axis and its underlying mechanisms to ASO pathology.

Keywords: ASO, HUVECs, miR-210, JAK1, STAT3, apoptosis 


\section{Introduction}

Atherosclerosis generally occurs when plaque builds up and hardens the arterial wall, due to the convergence of extracellular matrix, cholesterol, and cells in large quantities [1]. Atherosclerotic lesions are an important cause of morbidity and mortality as they reduce blood flow after years of slow disease progression [2]. Arteriosclerosis obliterans (ASO) is mainly treated with immediate-efficacy endovascular procedures, such as balloon angioplasty and stent implantation [3]. However, within 12 months of balloon angioplasty, stenosis recurrence and re-narrowed blood vessels are seen in $30-50 \%$ of patients. Approximately $12 \%$ of patients have severe ischemia and eventually require limb amputation [4]. ASO develops via several mechanisms and is influenced by multiple risk factors (age, male gender, smoking, hypertension, hyperlipidemia, diabetes mellitus, chronic renal failure, and hyperhomocysteinemia). During the development of ASO, endothelial dysfunction occurs, and macrophages and vascular smooth muscle cells (VSMCs) accumulate and produce TNF- $\alpha$, IL-6, and other pro-inflammatory cytokines [5]. Endothelial cells maintain both the integrity of blood vessels and the stability of the entire vascular system [6]. Endothelial dysfunction, largely attributed to apoptosis and inflammation [7], is one of the initial steps in the development of ASO and it plays a major role in its progression. Quiescent VSMCs migrate from the medial arterial wall into the intimal space and gradually form a neointima, which, combined with the presence of multiple extracellular matrix proteins, results in vascular stenosis. Therefore, the dysfunction of endothelial cells and VSMCs, including abnormal proliferation, migration, 
and apoptosis, are pivotal events resulting in arteriosclerosis. Thus, endothelial cells and VSMCs are crucial targets of ASO treatment.

MicroRNAs (miRNAs) are a class of noncoding RNAs that are highly conserved across species. miRNAs post-transcriptionally repress their target mRNAs that participate in diverse cellular functions [8-10]. miRNAs are involved in the onset and progression of ASO by regulating the function of VSMCs, macrophages, and endothelial cells [11-13]. The roles of $m i R-21, m i R-143$, and $m i R-221$ in the pathogenesis of arterial occlusive disease have previously been reported [14-16]. In cultured human arterial smooth muscle cells, $m i R-21$ decreases cell proliferation and migration by regulating tropomyosin 1 [17]. $m i R-143$ is required for Jag-1/Notch-induced VSMC differentiation [18]. miR-221 has pro-proliferation, pro-migration, and anti-apoptosis effects in VSMCs, but anti-proliferation, anti-migration, and pro-apoptosis effects in endothelial cells [16]. $m i R-210-5 p(m i R-210)$ acts as a tumor suppressor in ovarian cancer [19] and esophageal squamous cell carcinoma [20], by inhibiting cell proliferation and promoting apoptosis. miR-210 also suppresses human retinal endothelial cell migration and tube formation [21]. Higher levels of $m i R-210$ expression are observed in preeclamptic placentas [22], causing the suppression of trophoblast invasion. Furthermore, $m i R-210$ levels are significantly upregulated in ASO [23], but the detailed mechanism is not known.

The Janus kinase (JAK)-signal transducer and activator of transcription (STAT) pathway, an important oncogenic signaling pathway, is often activated in human malignant neoplasms [24]. It is activated by JAK tyrosine kinase, after ligand binding to a 
cell-surface receptor. STATs are transcription factors that are latent in the cytoplasm. After translocating to the nucleus, they bind to specific DNA elements to direct gene transcription, thus implying that STATs are phosphorylated by kinases. Previous studies have shown that JAK-STAT signaling is restricted to cytokine receptors and growth-factor receptors [25]. Activated STATs are involved in proliferation, cell death, and other normal cellular events [26]. Growing evidence suggests that STATs, especially STAT3, play pivotal roles in apoptosis [27]. However, reports regarding the role of the JAK-STAT signaling pathway in ASO are rare.

The aim of the present study was to evaluate the correlation between $m i R-210$ levels and vascular endothelial cell apoptosis in ASO and to investigate the molecular mechanism of $m i R-210$ expression and its relation to the JAK-STAT signaling pathway in ASO.

\section{Material and Methods}

\section{Clinical specimens}

Serum samples were collected from 20 ASO patients treated in the Department of Cardiology, Ningbo Huamei Hospital, Ningbo, China, from February 2016 to December 2018 and from 20 healthy volunteers. Intermittent claudication and ischemic rest pain, two major symptoms of ASO, were used as the main diagnostic criteria. Intermittent claudication is pain or fatigue that occurs in a muscle or muscle group on repetitive use. Ischemic rest pain indicates an advanced stage of the disease. Several other tests, such as 
angiography and measurements of ankle/brachial index and pulse wave velocity, were used as complementary parameters for ASO diagnosis. The Fontaine classification system was used to determine the stage of ischemia in the extremities. All ASO patients had characteristic symptoms of chronic limb ischemia, intermittent claudication, rest pain, or non-healing ischemic ulcers (Fontaine I, $n=5$; Fontaine II, $n=11$; Fontaine III, $n=4$ ), as confirmed by angiography. Baseline data for all patients are shown in Table 1. Individuals with none of these symptoms were recruited as non-ASO controls. Written informed consent was obtained from all participants and approval was obtained from the Ethics Review Committee of Ningbo Huamei Hospital.

\section{Animals}

Nondiabetic male B6 apolipoprotein E-deficient $\left(\mathrm{ApoE}^{--}\right)$mice (5 weeks old, 25-35 g), bred into a > 99\% C57BL/6J background, were supplied by Beijing Vital River Laboratory Animal Technology Co., Ltd (Beijing, China). Mice were fed a Western-type diet (21\% fat, $0.2 \%$ cholesterol) and water to induce ASO and were maintained on a $12 \mathrm{~h}$ light-dark cycle. After 8 weeks, the mice were bled to death under $20 \%$ urethane anesthesia. The study conformed to the laboratory animal guidelines ratified by the Animal Ethics Committee of Ningbo Huamei Hospital.

\section{Cell culture}

HUVECs, provided by the Shanghai Institute of Biochemistry and Cell Biology (Shanghai, China), were cultured in RPMI 1640 medium with $10 \%$ fetal bovine serum (FBS). The 
medium was replaced every 2 days, and cells were passaged after reaching 80-90\% confluence. To model arteriosclerosis, HUVECs were treated with $75 \mu \mathrm{g} / \mathrm{mL}$ oxidized low-density lipoprotein and $20 \mathrm{ng} / \mathrm{mL}$ platelet-derived growth factor-BB (Hangzhou Union Biotechnology Co., Ltd.) for $24 \mathrm{~h}$ at $37^{\circ} \mathrm{C}$ [28-30].

\section{Cell transfections}

An miR-210 mimic/inhibitor or corresponding negative control (NC) RNAs (GenePharma, Shanghai, China) were transfected into HUVECs using Lipofectamine 2000 (Invitrogen, Carlsbad, CA, USA). The following sequences were used: $m i R-210$ mimic, 5'-CUGUGC GUGUGACAGCGGCUGA-3'; NC mimic.

5'-UCACAACCUCCUAGAAAGAGUAGA-3'; miR-210 inhibitor,

5'-CUGUGCGUGUGACAGCGGCUGA-3'; NC inhibitor,

\section{5'-UUGUACUACACAAAAGUACUG-3'.}

\section{Cell survival assay}

A methyl thiazolyl tetrazolium (MTT) assay was used to investigate HUVEC survival. Briefly, cells were cultured in 96-well plates at a concentration of $4 \times 10^{3}$ cells/well and were then transfected with different RNAs. After reaching approximately $80 \%$ confluence, $20 \mu \mathrm{L}$ of MTT solution ( $0.5 \mathrm{mg} / \mathrm{mL}$; Sigma-Aldrich, St. Louis, MO, USA) was added to each well and cells were incubated for $4 \mathrm{~h}$. DMSO $(150 \mu \mathrm{L})$ was then added to each well to dissolve the resulting methyl crystals. Finally, optical density was measured at $540 \mathrm{~nm}$ using an Infinite M200 microplate reader (Tecan, Männedorf, Switzerland). 


\section{Colony-formation assay}

HUVECs were transfected with different RNAs 2 days prior to resuspension in RPMI 1640 medium containing 10\% FBS. Cells were subsequently plated on the top layer of base agar $(0.4 \%, 8 \mathrm{~mm})$ and transferred to 12 -well plates with a bottom layer of agar $(0.5 \%, 0.5 \mathrm{~mL})$. Four randomly chosen colonies on each plate were quantified after 2 weeks.

\section{Flow cytometry}

To analyze apoptosis, flow cytometry was performed after annexin V-fluorescein isothiocyanate (FITC) and propidium iodide (PI) staining, as previously reported [31-33]. Transfected cells $\left(5 \times 10^{4}\right)$ were resuspended in $20 \mu \mathrm{L}$ of annexin V-binding buffer $(10$ mM HEPES, pH 7.4; $140 \mathrm{mM} \mathrm{NaCl}$; and $2.5 \mathrm{mM} \mathrm{CaCl}_{2}$ ) and mixed with $10 \mu \mathrm{L}$ of FITC-conjugated anti-annexin V antibody (BD Biosciences, Franklin Lakes, NJ, USA). The mixture was then incubated in the dark at room temperature for 20 min and PI ( $5 \mu \mathrm{L})$ was added to the cell suspension immediately prior to analysis. Finally, the relative number of annexin V-and PI-positive cells was determined using a BD FACSVerse ${ }^{\mathrm{TM}}$ flow cytometer (BD Biosciences).

\section{Western blotting analysis}

Tris- $\mathrm{HCl}$ (50 mM) was mixed with sodium chloride (150 mM), NP-40 (1\%), and sodium dodecyl sulfonate $(0.1 \%)$ to prepare RIPA buffer $(\mathrm{pH} 7.2)$. The resulting buffer, along with a protease inhibitor solution (Roche, Basel, Switzerland) was used to lyse cells. Proteins 
were quantified using the Pierce ${ }^{\mathrm{TM}}$ BCA Protein Assay Kit (23225; Thermo Fisher Scientific, Waltham, MA, USA). After performing SDS-PAGE on $10 \%$ polyacrylamide gels (Bio-Rad, Hercules, CA, USA), the separated proteins were transferred to polyvinylidene difluoride (PVDF) membranes $(0.45 \mu \mathrm{m}$; Millipore, Burlington, MA, USA). The PVDF membranes were incubated overnight at $4{ }^{\circ} \mathrm{C}$ with primary antibodies (Abcam Cambridge, UK) against caspase-3 (1:2,000; ab4051), cleaved caspase-3 (1:500, ab2302), Bax (1:5,000, ab53154), Bcl-2 (1:5,000, ab59348), Janus kinase 1 (JAK1, 1:1,000, ab47435), STAT3 (1:1,000, ab5073), phosphorylated STAT3 (1:500, ab30647), and GAPDH (1:5,000, ab8245) and subsequently, with secondary antibodies. Immunoreactivity was detected using the West Femto Maximum Sensitivity Substrate Kit (Thermo Fisher Scientific) and a C-DiGit blot scanner (LI-COR Biosciences, Lincoln, NE, USA).

\section{RNA isolation and quantitative PCR}

Total RNA was extracted from cells using TRIzol (Invitrogen). miRNA was quantified in total RNA samples using SYBR Green (Invitrogen) and a LightCycler ${ }^{\circledR} 480$ Real-Time PCR System (Roche). GAPDH served as an internal reference. Quantitative PCR was performed in $20 \mu \mathrm{L}$ using a SYBR ${ }^{\circledR}$ Green PCR Master Mix. The internal reference was normalized relative to the calibrator (mean of the internal controls) to calculate the target values, using the $2^{-\Delta \Delta \mathrm{CT}}$ method.

\section{Dual-luciferase reporter assay}


The entire 3'-untranslated region (UTR) region of JAK1 was amplified and subcloned downstream of the GV126 luciferase gene. Following the prediction of miR-210-binding sites in the JAK1 gene, site-directed mutagenesis was performed to eliminate these binding sites. Cells expressing the mutant JAK1 3'-UTR region served as controls. The Renilla luciferase plasmid harboring the thymidine kinase promoter (TaKaRa, Kyoto, Japan) served as a reporter control to monitor transfection efficiency. The $m i R-210 / \mathrm{NC}$ mimic and wild-type/mutant $J A K 1$ were transfected with the reporter vector into HEK293T cells, after which a dual-luciferase reporter assay (DLRA) was performed. Cells were cultivated in DMEM supplemented with $10 \%$ FBS and mycillin and were incubated at $37^{\circ} \mathrm{C}$ under $5 \% \mathrm{CO}_{2}$. DMEM was replaced every 2 days. Between passages, the cells were digested with tryptase $(0.25 \%)$ and subcultured upon reaching $70-80 \%$ confluence.

\section{Immunofluorescence and confocal microscopy}

Immunofluorescence (IF) assays were performed $16 \mathrm{~h}$ after cell seeding, which began when cells reached $50 \%$ confluence. After fixing and permeabilizing cells in $100 \%$ methanol at room temperature for $15 \mathrm{~min}$, they were rinsed several times with PBS and rehydrated. PBS containing 1\% BSA was added to the rehydrated cells to block nonspecific binding. Next, the cells were rinsed thrice with PBS before performing the IF assay using DyLight 594- or FITC-conjugated secondary antibodies (1:100), with incubation at $4{ }^{\circ} \mathrm{C}$ for $16 \mathrm{~h}$. Nuclei were counterstained with DAPI (Invitrogen) and viewed under a TCS SP5 confocal laser-scanning microscope (Leica, Wetzlar, Germany). 


\section{JAK1 and STAT3 inhibitor treatment}

The JAK1 inhibitor, baricitinib (S2851; Selleck Chemicals, Houston, TX, USA) and the STAT3 inhibitor, Stattic (10 mM in DMSO, S7024, Selleckchem) were used to block JAK1-STAT3 signal transduction. After transfection of the $m i R-210 / \mathrm{NC}$ inhibitor for $32 \mathrm{~h}$, cells were incubated with either $2 \mu \mathrm{M}$ baricitinib or $10 \mu \mathrm{M}$ Stattic for 1 or $4 \mathrm{~h}$, respectively.

\section{Statistical analysis}

Data are expressed as mean \pm standard deviation and were analyzed using a one-way ANOVA or a two-tailed student's t-test. Statistical significance was set at $P<0.05$.

\section{Results}

$m i R-210$ was expressed at high levels in ASO patients

To determine the effect of $m i R-210$ on ASO, its serum levels were first measured in ASO patients. Compared with healthy volunteers, patients showed high levels of miR-210 (Figure 1A). Additionally, $m i R-210$ expression levels were found to be higher in atherosclerotic plaques of $\mathrm{ApoE}^{-/-}$mice than in normal congenic mice (Figure 1B). These data suggested that $m i R-210$ was involved in ASO pathogenesis.

\section{Regulated expression of miR-210-mediated VEC proliferation and apoptosis}


The effect of $m i R-210$ on HUVECs was subsequently investigated. To upregulate or downregulate $m i R-210$, HUVECs were transfected with an $m i R-210$ mimic or inhibitor, respectively. Quantitative reverse transcription PCR (qRT-PCR) results indicated that mimic or inhibitor transfection led to a significant increase or decrease in $m i R-210$ levels, respectively, in HUVECs (Figure 2A). The MTT assay showed a significant reduction in HUVEC proliferation 24-72 h post-transfection with the $m i R-210$ inhibitor, in comparison to those transfected with the NC inhibitor. However, HUVEC proliferation increased after transfection with the $m i R-210$ mimic (Figure 2B). As shown in the colony-formation assay, miR-210 downregulation reduced the number of HUVEC colonies, while cells transfected with the mimic produced more colonies (Figure 2C).

To further determine the effect of $m i R-210$ on apoptosis, transfected HUVECs were subsequently stained with annexin V-FITC/PI. Thirty-six hours after transfection, fewer apoptotic cells were observed in the $m i R-210$ mimic-transfected group, whereas a greater number of apoptotic cells was observed in the $m i R-210$ inhibitor-transfected group, compared with the NC groups (Figures 3A, 3B). Previous studies have shown that Bcl-2 increases cell survival, but Bax promotes apoptosis [34]. qRT-PCR results showed that the miR-210 mimic increased $B c l-2$ expression, but decreased Bax and caspase-3 expression and levels and caspase-3 cleavage (Figure 3C). Meanwhile, the miR-210 inhibitor decreased $B c l-2$ levels, but increased Bax and caspase-3 levels and caspase-3 cleavage, compared with the NC group (Figure 3D), indicating that $m i R-210$ promoted cell viability by reducing apoptosis. 
miR-210 targeted the $3^{\prime}$-UTR of JAK1

We subsequently used bioinformatics analysis to predict potential $m i R-210$ targets. As shown in Figure 4A, miR-210 may target JAK1. DLRA was performed to evaluate the direct interaction between $m i R-210$ and JAK1. Based on our data, the miR-210 mimic transfected HUVEC-interdicted luciferase to function and were fused by $75 \%$ with the 3'-UTR of JAK1 gene, in contrast with the other controls (Figure 4B).

Therefore, we first detected JAK1 expression in ASO samples and healthy controls. JAK1 mRNA levels were found to be lower in the 20 ASO samples than the healthy specimens (Figure 4C). Meanwhile, the expression levels of JAK1 mRNA and protein were lower in atherosclerotic plaques of $\mathrm{ApoE}^{-/-}$mice than in normal congenic mice, as detected by qRT-PCR and western blotting (Figures 4D, 4E).

The JAK-STAT pathway is well known for its involvement in processes such as cell division, cellular immunity, necrobiosis, and tumor formation [24]. Expression and phosphorylation JAK1 and STAT1/3 were reduced after transfection with the miR-210 mimic, whereas they were increased in cells transfected with the $m i R-210$ inhibitor (Figures 5A, 5B). Furthermore, IF analysis showed that miR-210 mimic transfection blocked STAT3 nuclear translocation, while $m i R$-210 inhibition increased nuclear STAT3 levels (Figures 5C, 5D). Hence, the 3'-UTR of JAK1 may be a target region of miR-210.

\section{The JAK1-STAT3 signaling axis was responsible for miR-210-mediated HUVEC} proliferation and apoptosis 
The role of the JAK1-STAT3 axis in mediating the effects of $m i R-210$ was determined by treating miR-210 inhibitor-transfected HUVECs with the JAK1 inhibitor, baricitinib and the STAT3 inhibitor, Stattic. Western blotting analysis showed that baricitinib treatment downregulated the expression and phosphorylation of STAT3, while Stattic treatment downregulated STAT3 phosphorylation without affecting its expression (Figures 6A, 6B). IF analysis showed that $m i R-210$ inhibition led to the nuclear translocation of STAT3, but this was inhibited by treatment with both baricitinib and Stattic (Figure 6C), indicating that the JAK1 and STAT3 pathways were blocked after drug treatment.

MTT assays were subsequently performed. These data indicated that baricitinib and Stattic restored the proliferation of HUVECs that was attenuated by $m i R-210$ inhibition (Figure 6D). Increased cell death was observed after $m i R-210$ inhibition, while cells incubated with baricitinib and Stattic had significantly higher cell survival rates (Figure 6E). Thus, blocking the JAK1-STAT3 signaling axis was sufficient to restore proliferation and suppress apoptosis induced via $m i R-210$ inhibition.

\section{Discussion}

As is well documented, miRNAs predominantly regulate target gene expression at the post-transcriptional level and they are crucial for mediating physiological and pathological processes, such as cancer [35], arthritis [36], and ASO [37, 38]. In ASO patients, serum $m i R-210$ levels were upregulated. Additionally, our data demonstrated that $m i R-210$ 
promoted the proliferation and inhibited the apoptosis of HUVECs via a mechanism that likely involved the regulation of JAK1 expression and JAK1-STAT3 signal transduction. Although a previous study showed miR-210 upregulation in the serum of ASO patients [39], the functions of $m i R-210$ in HUVECs have not previously been reported. In this study, we describe the roles of $m i R-210$ in ASO HUVECs. Our findings were similar to those of Zheng et al. [40], who found that $m i R-210$ overexpression significantly upregulates VEGF expression and promotes the proliferation, migration, and invasion of HUVECs. In the present study, cell proliferation was increased by $m i R-210$ in ASO HUVECs. Lu et al. demonstrated higher $m i R-210$ expression levels in oxygen-glucose-deprived endothelial progenitor cells. Under both normal and oxygen-glucose-deprived conditions, $m i R-210$ affected migration, proliferation, and tube formation [41].

Activated STAT3 serves as an important biological marker that mediates VEGF-induced activation of endothelial cells [42-44]. There is potential for the development of antiangiogenic therapy targeting the STAT3 signaling pathway that is mediated by VEGF/VEGFR. However, as STAT signaling status differs in different endothelial cells, it is unclear whether STAT3 is activated in HUVECs of ASO patients [45]. Here, a DLRA was used to show that $m i R-210$ directly targeted JAK1. miR-210 inhibition has been suggested to promote JAK1-STAT3 activity and transduction, to repress VEC proliferation, and the inhibition of both JAK1 and STAT3 has been shown to reverse these effects. Indeed, we demonstrated that baricitinib and Stattic treatment efficiently blocked $m i R-21$ 
inhibition-induced STAT3 phosphorylation, with significantly less HUVEC apoptosis and restored HUVEC proliferation. In agreement with our findings, recent studies have demonstrated that JAK or STAT inhibitors decrease apoptosis, and STAT upregulation promotes apoptosis in multiple cell lines, such as advanced ovarian clear cell carcinoma [46], myocardial microvascular endothelial [47], and osteosarcoma cells [48].

\section{Conclusions}

In conclusion, the present study demonstrated that $m i R-210$ levels are significantly increased in ASO serum samples. In addition, $m i R-210$ promoted the proliferation of VECs, but its inhibition repressed proliferation, at least to some extent, when JAK1 was directly targeted by miR-210. Meanwhile, activation of downstream STAT1, STAT3, and SOCS1 were also inhibited by miR-210. This study identified a potential therapeutic target, miR-210, for ASO.

\section{Acknowledgments}

None. 


\section{References}

[1] Fuster V, Badimon L, Badimon J J and Chesebro J H. The pathogenesis of coronary artery disease and the acute coronary syndromes. New England journal of medicine 1992; 326: 310-318.

[2] Ross R. The pathogenesis of atherosclerosis: a perspective for the 1990s. Nature 1993; 362: 801 .

[3] Malgor R D, Alalahdab F, Elraiyah T A, Rizvi A Z, Lane M A, Prokop L J, et al. A systematic review of treatment of intermittent claudication in the lower extremities. Journal of vascular surgery $2015 ; 61: 54$ S-73S.

[4] Reinecke H, Unrath M, Freisinger E, Bunzemeier H, Meyborg M, Lüders F, et al. Peripheral arterial disease and critical limb ischaemia: still poor outcomes and lack of guideline adherence. European heart journal 2015; 36: 932-938.

[5] Musialik K, Szulinska M, Hen K, Skrypnik D and Bogdanski P. The relation between osteoprotegerin, inflammatory processes, and atherosclerosis in patients with metabolic syndrome. Eur. Rev. Med. Pharmacol. Sci 2017; 21: 4379-4385.

[6] Cao Y, Liu D and Zhang H. MiR-7 regulates the PI3K/AKT/VEGF pathway of retinal capillary endothelial cell and retinal pericytes in diabetic rat model through IRS-1 and inhibits cell proliferation. European review for medical and pharmacological sciences 2018; 22: 4427-4430.

[7] Hou H-F, Yuan N, Guo Q, Sun T, Li C, Liu J-B, et al. Citreoviridin enhances atherogenesis in hypercholesterolemic ApoE-deficient mice via upregulating 
inflammation and endothelial dysfunction. PloS one 2015; 10: e0125956.

[8] Farh K K-H, Grimson A, Jan C, Lewis B P, Johnston W K, Lim L P, et al. The widespread impact of mammalian MicroRNAs on mRNA repression and evolution. Science 2005; 310: 1817-1821.

[9] Bartel D P. MicroRNAs: target recognition and regulatory functions. cell 2009; 136: 215-233.

[10]Zhao M, Wang K, Shang J, Liang Z, Zheng W and Gu J. MiR-345-5p inhibits tumorigenesis of papillary thyroid carcinoma by targeting SETD7. Archives of Medical Science: AMS 2020; 16: 888.

[11]Siasos G, Kollia C, Tsigkou V, Basdra E K, Lymperi M, Oikonomou E, et al. MicroRNAs: Novel diagnostic and prognostic biomarkers in atherosclerosis. Current topics in medicinal chemistry $2013 ; 13: 1503-1517$.

[12] Imanishi T and Akasaka T. MicroRNAs in peripheral artery disease. Current topics in medicinal chemistry 2013; 13: 1589-1595.

[13] Madrigal-Matute J, Rotllan N, Aranda J F and Fernández-Hernando C. MicroRNAs and atherosclerosis. Current atherosclerosis reports 2013; 15: 322.

[14]Wang M, Li W, Chang G-Q, Ye C-S, Ou J-S, Li X-X, et al. MicroRNA-21 regulates vascular smooth muscle cell function via targeting tropomyosin 1 in arteriosclerosis obliterans of lower extremities. Arteriosclerosis, thrombosis, and vascular biology 2011; 31: 2044-2053.

[15]Boucher J M, Peterson S M, Urs S, Zhang C and Liaw L. The miR-143/145 cluster is 
a novel transcriptional target of Jagged-1/Notch signaling in vascular smooth muscle cells. Journal of Biological Chemistry 2011; 286: 28312-28321.

[16]Liu X, Cheng Y, Yang J, Xu L and Zhang C. Cell-specific effects of miR-221/222 in vessels: molecular mechanism and therapeutic application. Journal of molecular and cellular cardiology 2012; 52: 245-255.

[17]Wang M, Li W, Chang G Q, Ye C S, Ou J S, Li X X, et al. MicroRNA-21 regulates vascular smooth muscle cell function via targeting tropomyosin 1 in arteriosclerosis obliterans of lower extremities. Arteriosclerosis, thrombosis, and vascular biology 2011; 31: 2044-2053.

[18]Boucher J M, Peterson S M, Urs S, Zhang C and Liaw L. The miR-143/145 cluster is a novel transcriptional target of Jagged-1/Notch signaling in vascular smooth muscle cells. The Journal of biological chemistry 2011; 286: 28312-28321.

[19] Giannakakis A, Sandaltzopoulos R, Greshock J, Liang S, Huang J, Hasegawa K, et al. miR-210 links hypoxia with cell cycle regulation and is deleted in human epithelial ovarian cancer. Cancer biology \& therapy 2008; 7: 255-264.

[20]Tsuchiya S, Fujiwara T, Sato F, Shimada Y, Tanaka E, Sakai Y, et al. MicroRNA-210 regulates cancer cell proliferation through targeting fibroblast growth factor receptor-like 1 (FGFRL1). Journal of Biological Chemistry 2011; 286: 420-428.

[21] Yang Q, Wang P, Du X, Wang W, Zhang T and Chen Y. Direct repression of IGF2 is implicated in the anti-angiogenic function of microRNA-210 in human retinal endothelial cells. Angiogenesis 2018; 21: 313-323. 
[22]Enquobahrie D A, Abetew D F, Sorensen T K, Willoughby D, Chidambaram K and Williams M A. Placental microRNA expression in pregnancies complicated by preeclampsia. American journal of obstetrics and gynecology 2011; 204: 178. e112-178. e121.

[23] Anton L, Olarerin-George A O, Schwartz N, Srinivas S, Bastek J, Hogenesch J B, et al. miR-210 inhibits trophoblast invasion and is a serum biomarker for preeclampsia. The American journal of pathology 2013; 183: 1437-1445.

[24] Yu H and Jove R. The STATs of cancer-new molecular targets come of age. Nature Reviews Cancer 2004; 4: 97.

[25]Darnell J E, Kerr I M and Stark G R. Jak-STAT pathways and transcriptional activation in response to IFNs and other extracellular signaling proteins. Science 1994; 264: $1415-1421$

[26]Burger M, Hartmann T, Burger J A and Schraufstatter I. KSHV-GPCR and CXCR2 transforming capacity and angiogenic responses are mediated through a JAK2-STAT3-dependent pathway. Oncogene 2005; 24: 2067.

[27]Fathi N, Rashidi G, Khodadadi A, Shahi S and Sharifi S. STAT3 and apoptosis challenges in cancer. International journal of biological macromolecules 2018; 117 : 993-1001.

[28]Zhu Y, Lin J H-C, Liao H-L, Friedli Jr O, Verna L, Marten N W, et al. LDL induces transcription factor activator protein-1 in human endothelial cells. Arteriosclerosis, thrombosis, and vascular biology 1998; 18: 473-480. 
[29]Chen Z, Wang M, He Q, Li Z, Zhao Y, Wang W, et al. MicroRNA-98 rescues proliferation and alleviates ox-LDL-induced apoptosis in HUVECs by targeting LOX-1. Experimental and therapeutic medicine 2017; 13: 1702-1710.

[30]Li X, Yao N, Zhang J and Liu Z. MicroRNA-125b is involved in atherosclerosis obliterans in vitro by targeting podocalyxin. Molecular medicine reports $2015 ; 12$ : $561-568$.

[31] Ying C, Wang S, Lu Y, Chen L, Mao Y, Ling H, et al. Glucose fluctuation increased mesangial cell apoptosis related to AKT signal pathway. Archives of medical science: AMS 2019; 15: 730 .

[32]Eken M K, Ersoy G S, Kaygusuz E I, Devranoğlu B, Takır M, Çilingir Ö T, et al. Etanercept protects ovarian reserve against ischemia/reperfusion injury in a rat model. Archives of Medical Science: AMS 2019; 15: 1104.

[33]Guo H, He Y, Bu C and Peng Z. Antitumor and apoptotic effects of 5-methoxypsoralen in U87MG human glioma cells and its effect on cell cycle, autophagy and PI3K/Akt signaling pathway. Archives of medical science: AMS 2019; 15: 1530 .

[34] Kulsoom B, Shamsi T S, Afsar N A, Memon Z, Ahmed N and Hasnain S N. Bax, $\mathrm{Bcl}-2$, and $\mathrm{Bax} / \mathrm{Bcl}-2$ as prognostic markers in acute myeloid leukemia: are we ready for Bcl-2-directed therapy? Cancer Manag Res 2018; 10: 403-416.

[35]Sun T, Wang Q, Balk S, Brown M, Lee G-S M and Kantoff P. The role of microRNA-221 and microRNA-222 in androgen-independent prostate cancer cell 
lines. Cancer research 2009; 69: 3356-3363.

[36]Furer V, Greenberg J D, Attur M, Abramson S B and Pillinger M H. The role of microRNA in rheumatoid arthritis and other autoimmune diseases. Clinical immunology 2010; 136: 1-15.

[37]Ju H, Venema V J, Marrero M B and Venema R C. Inhibitory interactions of the bradykinin B2 receptor with endothelial nitric-oxide synthase. Journal of Biological Chemistry 1998; 273: 24025-24029.

[38]Ji R, Cheng Y, Yue J, Yang J, Liu X, Chen H, et al. MicroRNA expression signature and antisense-mediated depletion reveal an essential role of MicroRNA in vascular neointimal lesion formation. Circulation research 2007; 100: 1579-1588.

[39]Li Y, Yang C, Zhang L and Yang P. MicroRNA-210 induces endothelial cell apoptosis by directly targeting PDK1 in the setting of atherosclerosis. Cellular \& molecular biology letters 2017; 22: 3 .

[40]Zheng Z, Liu L, Zhan Y, Yu S and Kang T. Adipose-derived stem cell-derived microvesicle-released miR-210 promoted proliferation, migration and invasion of endothelial cells by regulating RUNX3. Cell Cycle 2018; 17: 1026-1033.

[41]Lu W-J, Liang H-B, Li Y-F, Tu X-Q, He J-R, Ding K-Q, et al. MicroRNA-210-3p Targets RGMA to Enhance the Angiogenic Functions of Endothelial Progenitor Cells Under Hypoxic Conditions. Frontiers in cellular neuroscience 2019; 13:

[42]Chen Z and Han Z C. STAT3: a critical transcription activator in angiogenesis. Medicinal research reviews 2008; 28: 185-200. 
[43] Wei L-H, Kuo M-L, Chen C-A, Chou C-H, Lai K-B, Lee C-N, et al. Interleukin-6 promotes cervical tumor growth by VEGF-dependent angiogenesis via a STAT3 pathway. Oncogene 2003; 22: 1517.

[44] Yahata Y, Shirakata Y, Tokumaru S, Yamasaki K, Sayama K, Hanakawa Y, et al. Nuclear translocation of phosphorylated STAT3 is essential for vascular endothelial growth factor-induced human dermal microvascular endothelial cell migration and tube formation. Journal of Biological Chemistry 2003; 278: 40026-40031.

[45]Bartoli M, Platt D, Lemtalsi T, Gu X, Brooks S E, Marrero M B, et al. VEGF differentially activates STAT3 in microvascular endothelial cells. The FASEB journal 2003; 17: 1562-1564.

[46]Bixel K, Saini U, Kumar Bid H, Fowler J, Riley M, Wanner R, et al. Targeting STAT3 by HO3867 induces apoptosis in ovarian clear cell carcinoma. Int J Cancer 2017; 141: $1856-1866$.

[47]Cui Z T, Liu J P and Wei W L. The effects of tanshinone IIA on hypoxia/reoxygenation-induced myocardial microvascular endothelial cell apoptosis in rats via the JAK2/STAT3 signaling pathway. Biomed Pharmacother 2016; 83: $1116-1126$.

[48]Liu K, Ren T, Huang Y, Sun K, Bao X, Wang S, et al. Apatinib promotes autophagy and apoptosis through VEGFR2/STAT3/BCL-2 signaling in osteosarcoma. Cell Death Dis 2017; 8: e3015. 


\begin{tabular}{|l|l|l|l|}
\hline & ASO group & Control & p-value \\
& $(\mathrm{n}=20)$ & group & \\
\hline Males/females & $9 / 11$ & $8 / 12$ & 0.21 \\
\hline Age (years) & $49.5 \pm 5.6$ & $50.2 \pm 5.4$ & 0.45 \\
\hline Body mass index (kg/m2) & $27.6 \pm 3.5$ & $26.9 \pm 3.7$ & 0.43 \\
\hline Total cholesterol (mg/dl) & $202.3 \pm 45.9$ & $193.4 \pm 39.0$ & 0.10 \\
\hline HDL-C(mg/dl) & $51.2 \pm 13.1$ & $49.5 \pm 11.3$ & 0.23 \\
\hline LDL-C(mg/dl) & $114.16 \pm 36.2$ & $118.3 \pm 33.6$ & 0.32 \\
\hline Triglyceride (mg/dl) & $168.6 \pm 55.8$ & $153.6 \pm 59.7$ & 0.18 \\
\hline GFR(ml/min/1.73m $\left.{ }^{2}\right)$ & $111.5 \pm 15.1$ & $111.6 \pm 14.9$ & 0.52 \\
\hline Fasting blood-glucose(mmol/l) & $4.9 \pm 0.5$ & $5.0 \pm 0.5$ & 0.36 \\
\hline Heart rate(beats/minute) & $74.5 \pm 10.8$ & $74.8 \pm 9.8$ & 0.25 \\
\hline SBP(mm Hg) & $145.0 \pm 12.0$ & $138.5 \pm 15.6$ & 0.02 \\
\hline DBP(mm Hg) & $84.5 \pm 8.5$ & $83.4 \pm 9.1$ & 0.23 \\
\hline CRP(mg/l) & $6.8 \pm 2.5$ & 0.008 \\
\hline
\end{tabular}

Table 1. Demographic, clinic and laboratory characteristics between groups.

HDL-C, highdensity lipoprotein cholesterol; LDL-C, low density lipoprotein cholesterol; GFR, glomerular filtration rate; CRP, C-reactive protein; DBP, diastolic blood pressure; SBP, systolic blood pressure. Data are presented as the mean \pm standard deviation 
Figure legends

Figure 1. $m i R-210$ expression in ASO patients

(A) qRT-PCR was performed to analyze miR-210 expression in serum samples of 20 ASO patients and 20 healthy volunteers. (B) Expression levels of $m i R-210$ in $\mathrm{ApoE}^{-{ }^{--}}$mice with ASO were measured by qRT-PCR. Results are presented as mean \pm SD. $* * P<0.01$, *** $P$ $<0.001$.

\section{Figure 2. Effect of $m i R-210$ on HUVEC proliferation}

(A) HUVECs were transfected for $36 \mathrm{~h}$ with $m i R-210$ or negative control (NC)

mimic/inhibitor. $m i R-210$ expression levels were subsequently measured in human vascular endothelial cells (HUVECs) by qRT-PCR. (B) VEC proliferation rate was measured by MTT assay, 24, 48, and $72 \mathrm{~h}$ after transfection. (C) $m i R-210$ or NC mimic/inhibitor-transfected HUVECs underwent a colony-formation assay. The right panel shows the number of colonies formed in each group. Results are presented as mean $\pm \mathrm{SD} .{ }^{*} P<0.05, * * P<0.01$

\section{Figure 3. Effect of $m i R-210$ on HUVEC apoptosis}

(A, B) To determine the total number of apoptotic cells, HUVECs were stained with annexin V-FITC/PI for flow cytometry. In each plot, the right quadrant indicates apoptotic cells at an early stage. (C, D) Western blotting was performed to detect the levels of Bcl-2, Bax, caspase-3, and cleaved caspase-3 in HUVECs transfected with miR-210 or NC mimic/inhibitor. cCaspase-3, cleaved caspase-3. Data represent the mean $\pm \mathrm{SD}$. ${ }^{*} P<0.05$. 
Figure 4. JAK1 was directly targeted by $m i R-210$

(A) Graphic representation of conserved miR-210-binding motif in the JAK1 3'-UTR. Sequences complementary to the $m i R-210$ seed region are displayed. They correspond to wild-type (WT) and mutant (MU) sequences of the JAK1 3'-UTR. (B) Luciferase activity following the transfection of $m i R-210$ mimics with luciferase reporter constructs incorporating either WT or of MU JAK1 3'-UTR sequences. After normalization to $\beta$-galactosidase activity, luciferase activity of the WT JAK1 3'-UTR constructs were significantly reduced by miR-210 compared with MU JAK1 3'-UTR constructs. (C) qRT-PCR was performed to measure JAK1 mRNA levels in serum samples of $20 \mathrm{ASO}$ patients and 20 healthy volunteers. (D, E) JAK1 mRNA and protein expression levels were measured in ApoE-/- mice with ASO via qRT-PCR and western blotting, respectively. Data are presented as mean $\pm \mathrm{SD} .{ }^{*} P<0.05$.

\section{Figure 5. $m i R-210$ regulated JAK1-STAT3 pathway activation}

(A, B) Western blotting analysis was performed to determine JAK1 and STAT1/3 expression and phosphorylation levels in HUVECs transfected with miR-210 or negative control (NC) mimic/inhibitor. (C, D) Cellular localization of STAT3 in HUVECs transfected with $m i R-210$ or NC mimic/inhibitor was determined by immunofluorescence. pSTAT3, phosphorylated STAT3. Data are presented as mean \pm SD. $* P<0.05$.

Figure 6. JAK1 and STAT3 restored HUVEC proliferation impaired by $m i R-210$ inhibition 
VECs were initially transfected with either an $m i R-210$ inhibitor or negative control (NC) counterpart for $32 \mathrm{~h}$ before treatment with $2 \mu \mathrm{M}$ baricitinib for $1 \mathrm{~h}$ or $10 \mu \mathrm{M}$ Stattic for $4 \mathrm{~h}$. (A, B) Western blotting was performed to verify the efficacy of baricitinib and Stattic treatment on the levels of JAK1, STAT3, and phosphorylated STAT3 in HUVECs. (C) Immunofluorescence was performed to detect the nuclear translocation of STAT3 in HUVECs. (D) An MTT assay measured the effects of baricitinib and Static on HUVEC proliferation. (E) Apoptosis of transfected and treated HUVECs was evaluated by flow cytometry. pSTAT3, phosphorylated STAT3. Results are expressed as mean \pm SD. ${ }^{*} P<$ 0.05 . 


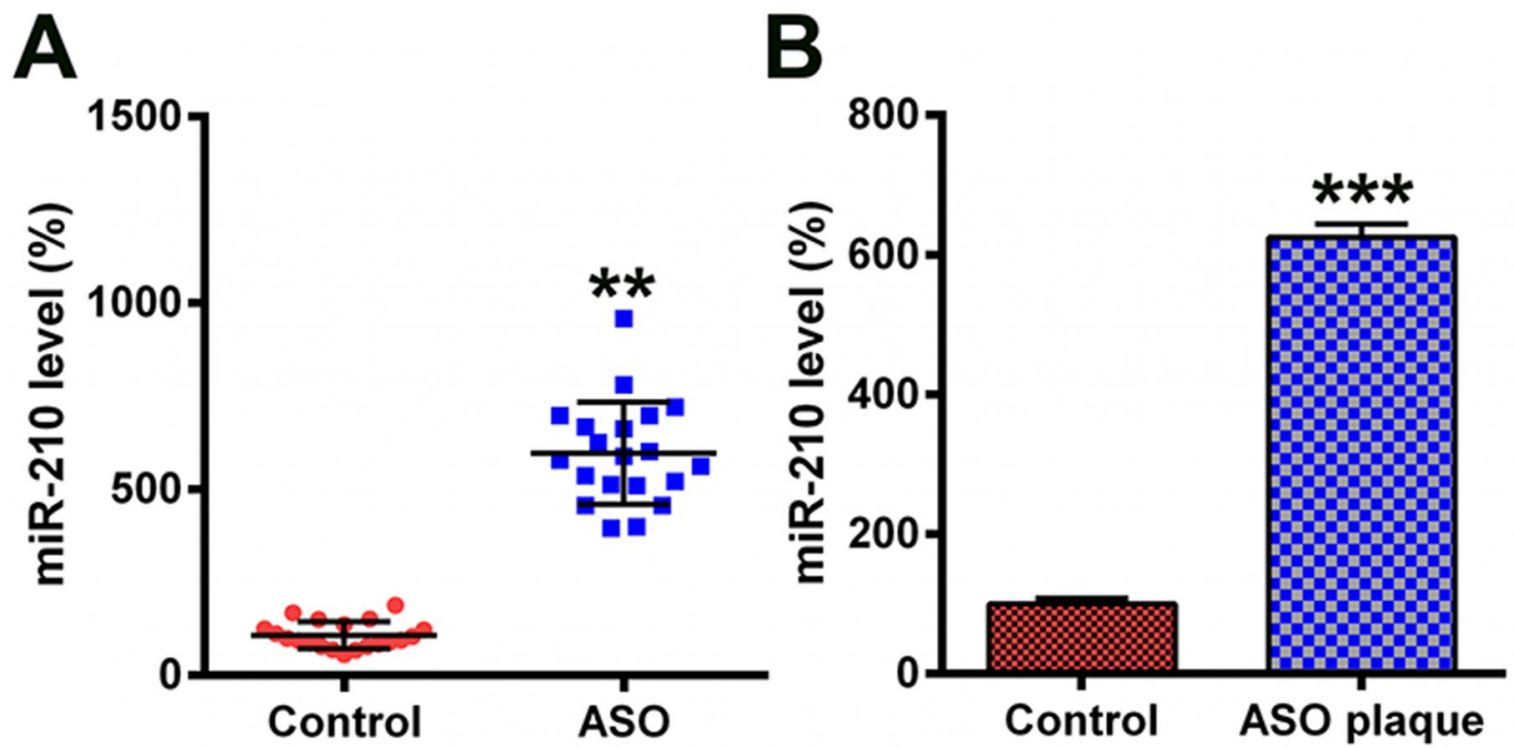


A

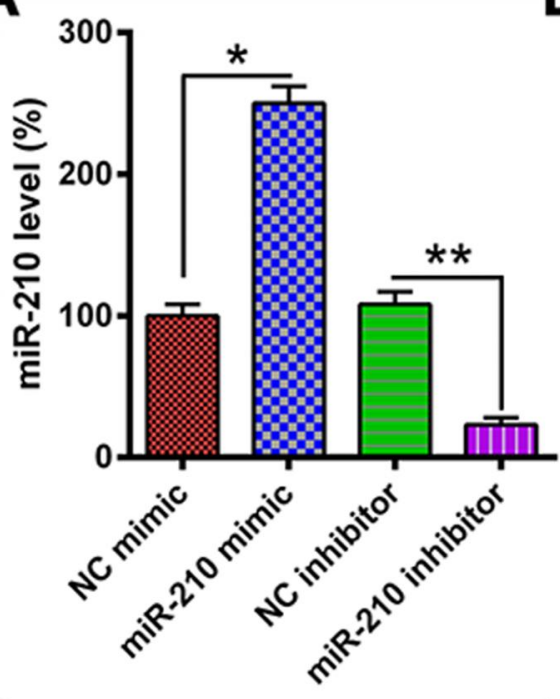

C

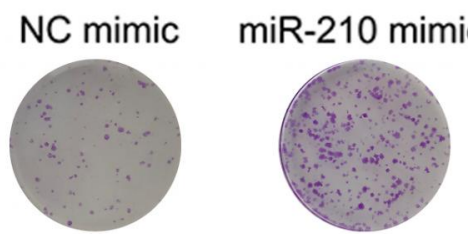

NC inhibitor miR-210 inhibitor

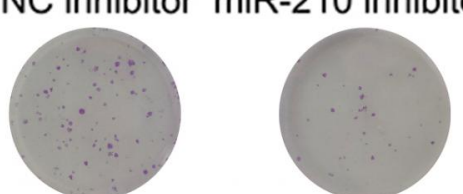

B

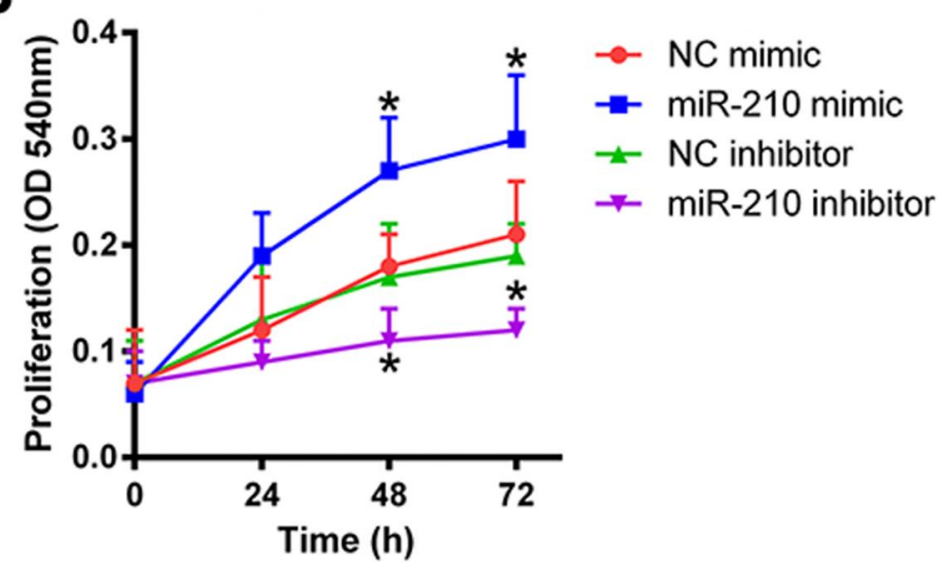

D

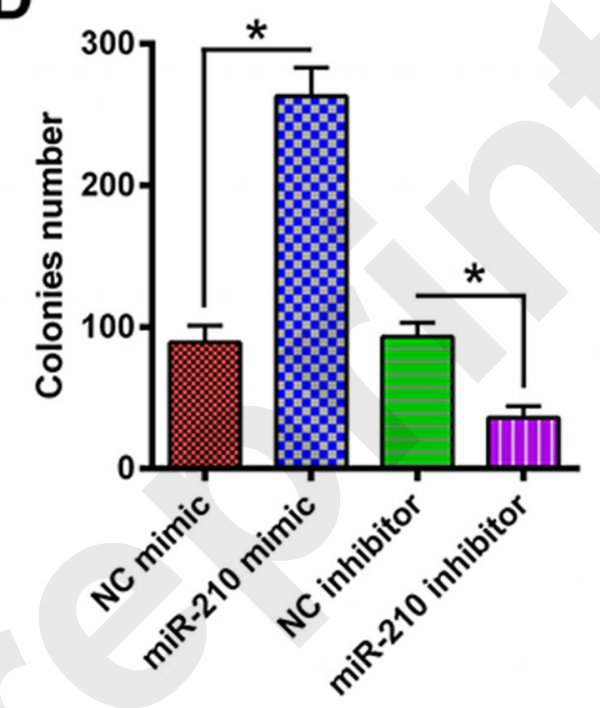




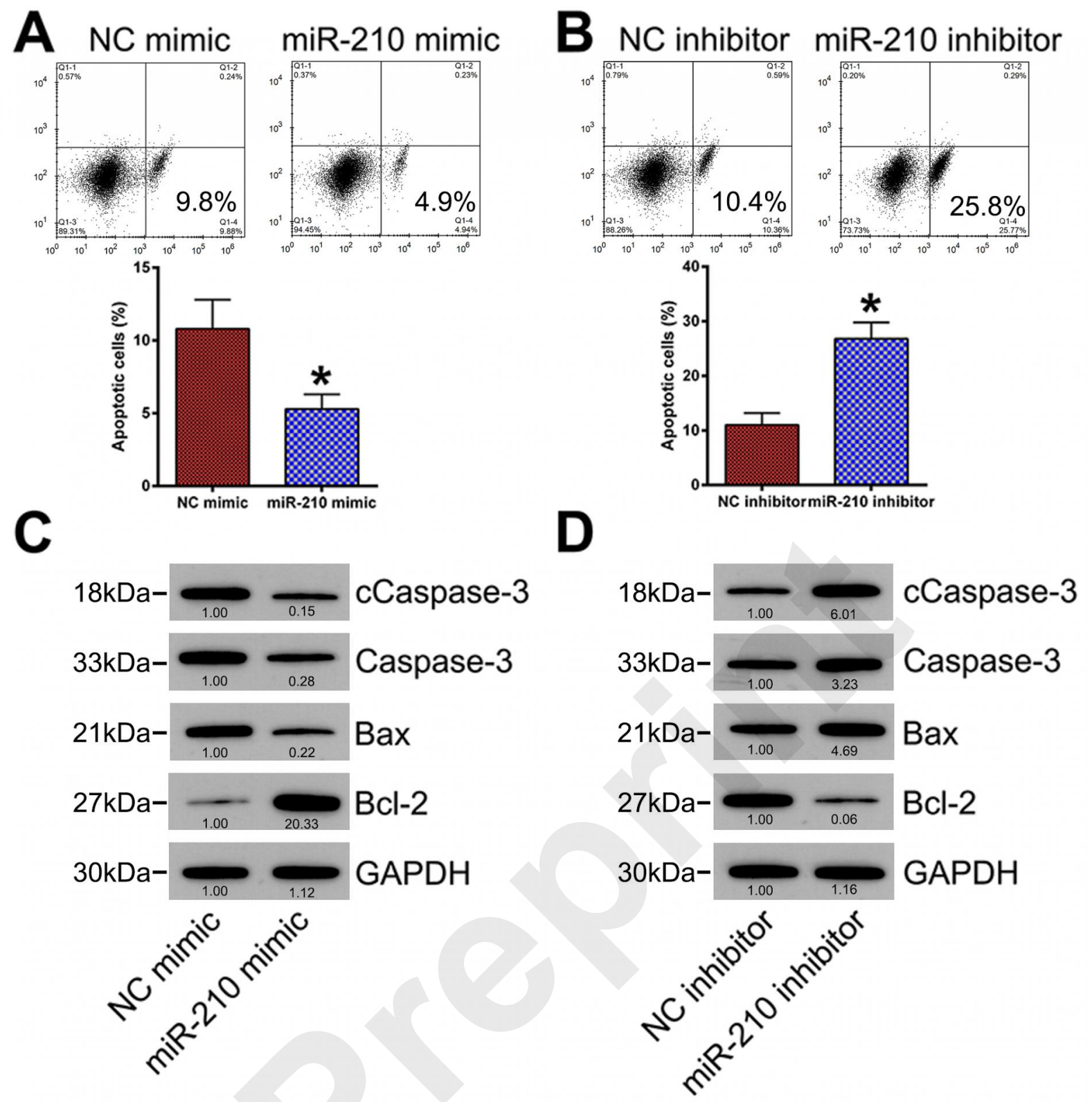


A
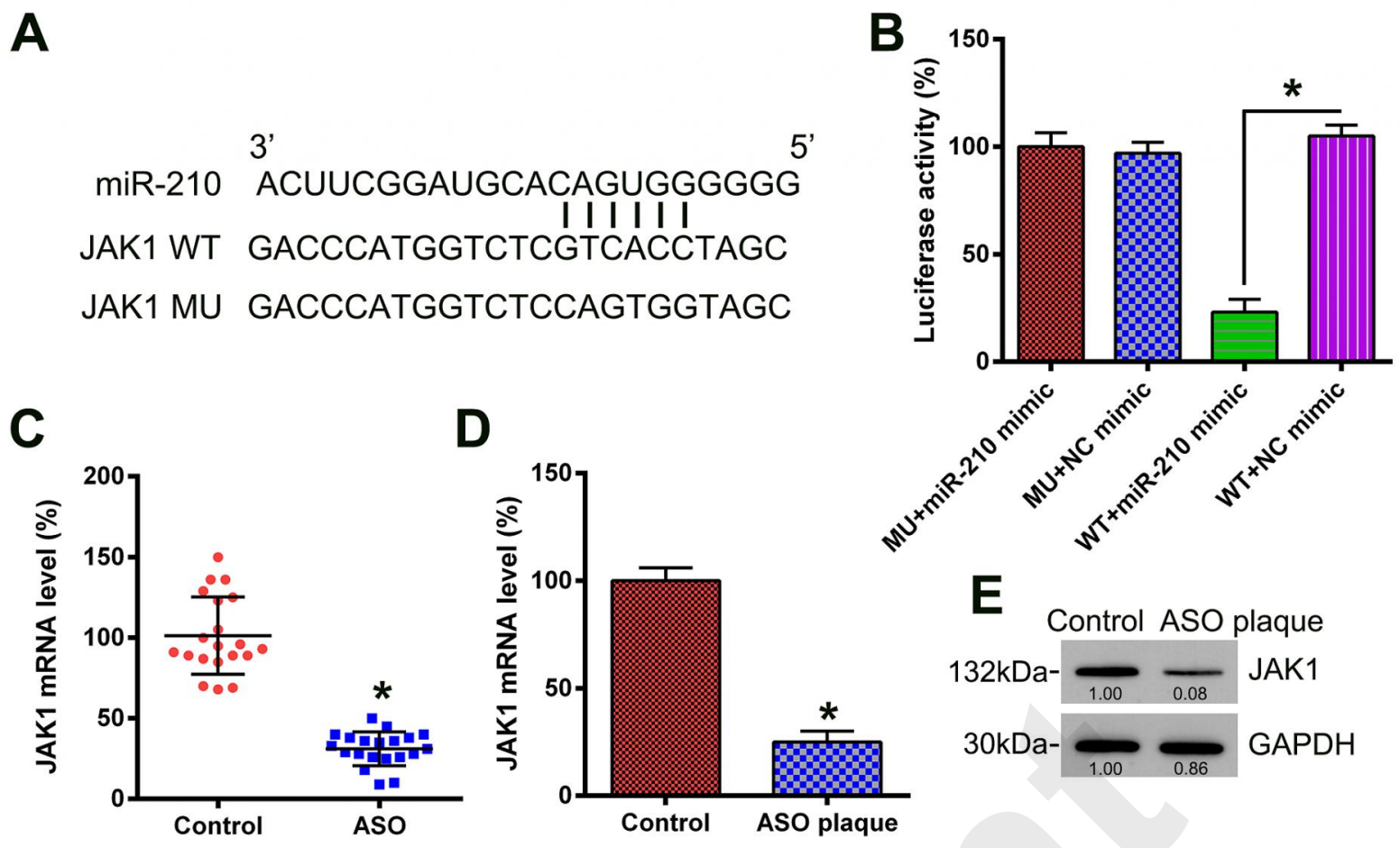

E

$132 \mathrm{kDa}-\underset{1.00}{-} \frac{}{0.08}$ JAK1

$30 \mathrm{kDa}-\underset{1.00}{\longrightarrow} \underset{0.86}{\mathrm{GAPDH}}$ 

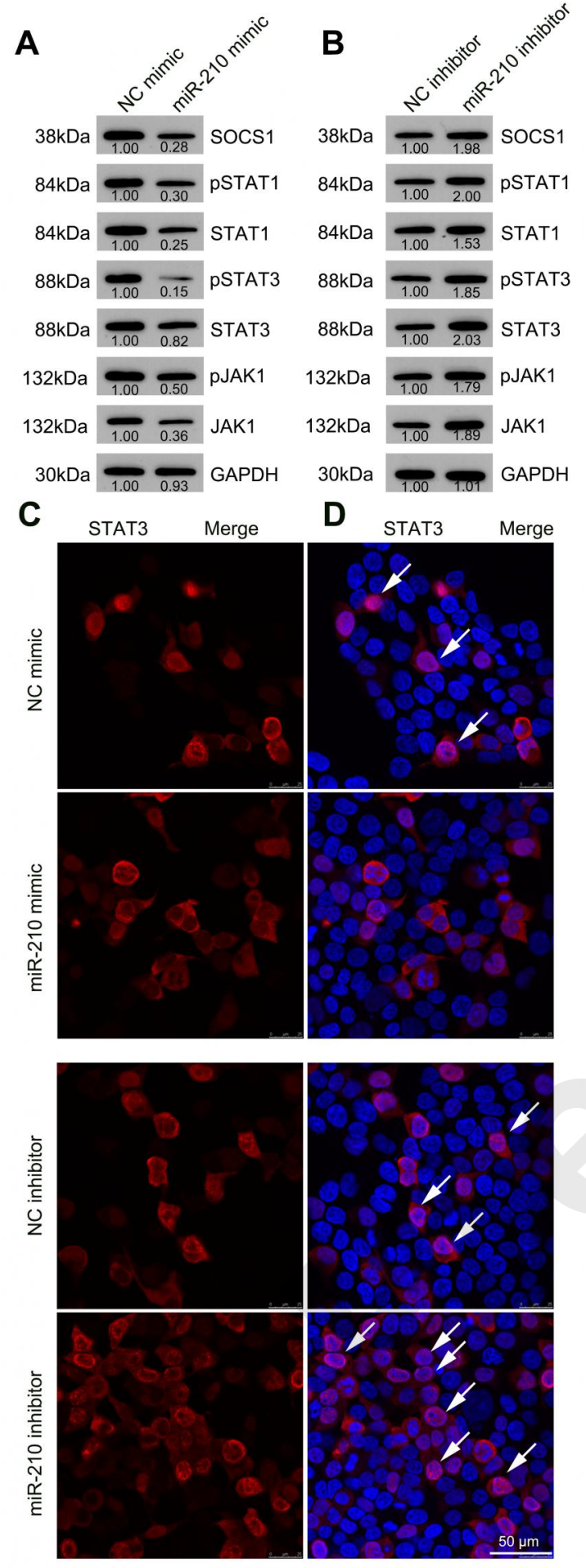

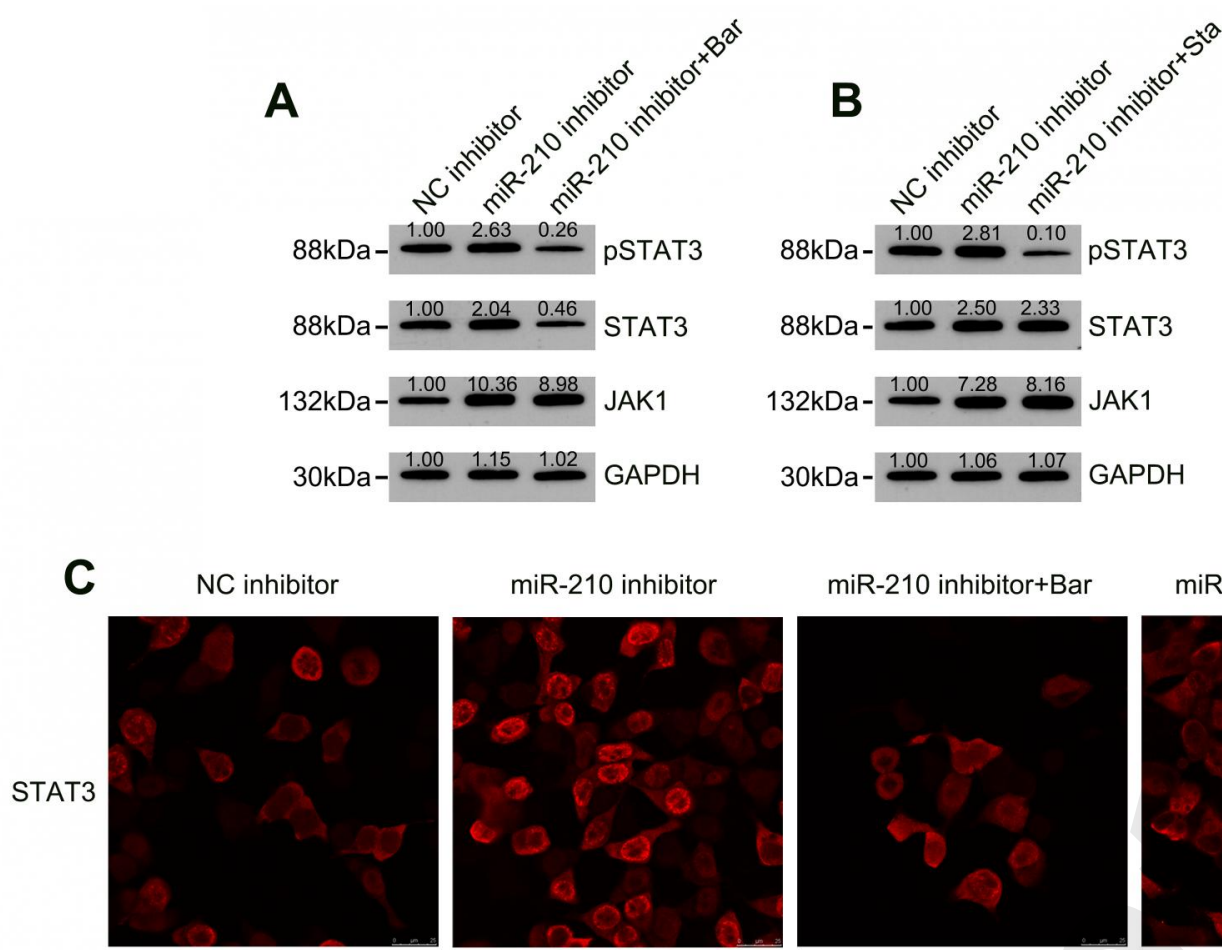

miR-210 inhibitor+Sta
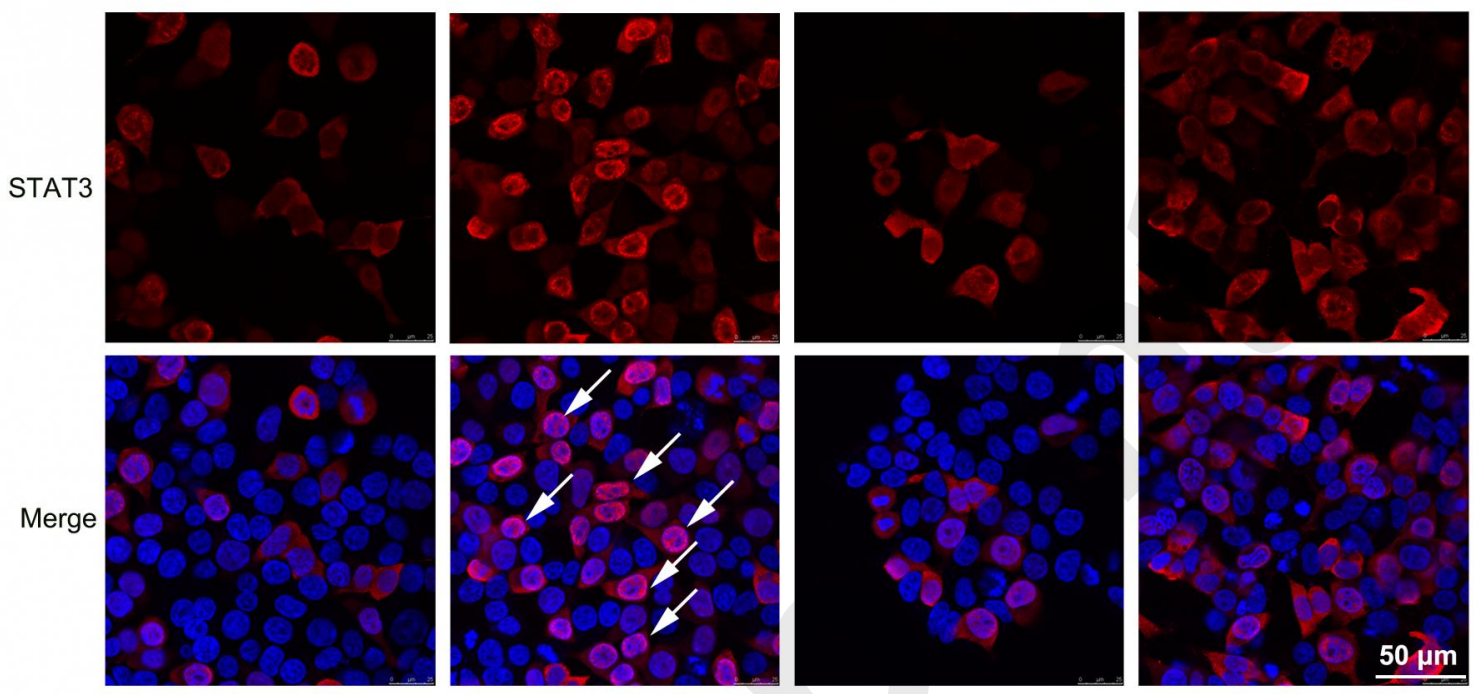

\section{D}

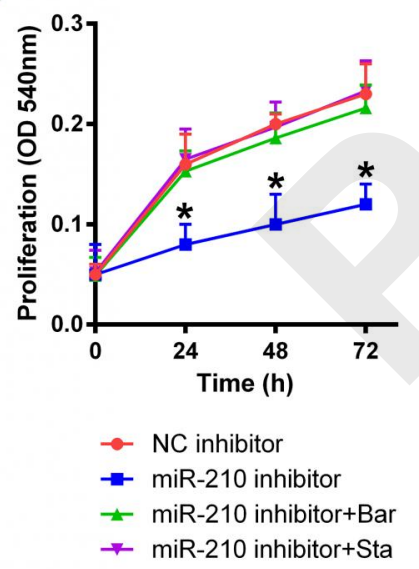

E

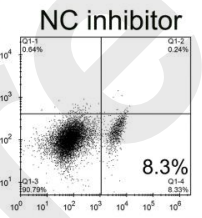

miR-210 inhibitor+Bar
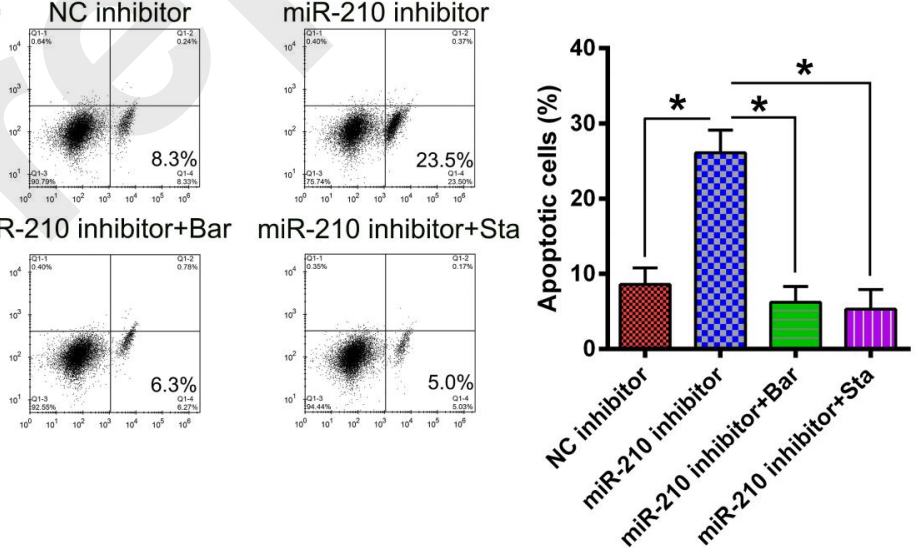